\title{
Visual and semantic factors in recognition from long-term memory
}

\author{
DOUGLAS J. HERMANN \\ Hamilton College, Clinton, New York 13323 \\ and \\ JOHN P. MCLAUGHLIN and BILLIE C. NELSON \\ University of Delaware, Newark, Delaware 19711
}

\begin{abstract}
In recognition tests, physical and semantic relationships between targets and distractors have been shown, in separate manipulations, to affect the latency of subject's decision. Recognition was tested for distractors which were visually similar or dissimilar to targets and which belonged to the target categories or to nontarget categories in order to examine the interaction of these dimensions. Rejection latency was longer for target category than for nontarget category distractors. Latency was also longer for visually similar than visually dissimilar distractors, but only when combined with target category probes. This interaction can be explained by the hypothesis that word recognition depends on the analysis of several dimensions of the probe stimulus, and rejection can occur before all such analyses have been completed.
\end{abstract}

Recent research has indicated that the recognition latency for targets from a well-memorized list and for distractors depends on both the physical aspects of test words and the meaning of test words. Juola, Fischler, Wood, and Atkinson (1971) required subjects to memorize a list of 16 unrelated words and then classify a series of single-word displays as containing either a memorized word, target, or a nonmemorized word, distractor. The set of distractors included words which were homophones of the targets and nonhomophones. Classification latency was slower to homophone distractors than to nonhomophones only if the homophones were visually similar to a target, e.g, SAIL and SALE. Items were considered dissimilar if two letters were different, e.g., CENTS or SENSE; or were different in length, e.g., SOME or SUM. Juola et al. suggested that visual similarity between target and distractor might lead to confusions, longer times to identify words, and identification errors, although the effect of visual information on initial familiarity judgments was not ruled out. Later Atkinson and Juola (Note 1) stated that visual similarity could affect the speed with which the appropriate lexical node (repository of codes used in handling information) is accessed. In either case, the suggestion was that visual similarity had its effects in an encoding stage prior to the evaluation of the probe's familiarity. A similar effect of expectancy leading to misreading errors was reported by Neisser and Beller (1965). They found that some subjects misread a distractor as a target when searching a list of words for the target. Targets in long-term memory

Thomas Duszak collected a portion of these data.

Reprint requests should be sent to John P. McLaughlin, Department of Psychology, University of Delaware, Newark, Delaware 19711. seem to affect the reading of probes.

Juola et al. also showed separately that latency varied with the meaning of distractor words. Latency was greater when distractors were synonyms of targets than when they were not. A similar effect of meaning on recognition latency has also been reported by Okada and Burrows (Note 2). They required subjects to memorize a list composed of examples from five taxonomic categories, e.g., animals, occupations. Subjects' latency was faster to distractors which belonged to categories not on the memorized list (nontarget categories) than to distractors drawn from the categories used on the memorized list (target categories).

While the above experiments show that recognition latency is sensitive to physical and semantic factors, the interrelationship of these factors remains to be elucidated. No experiment which varied physical and semantic factors orthogonally within the same task has been reported.

If recognition is viewed as a decision based on the outcome of analyses of a test item, then one might assume that rejection of a distractor would occur following an item's failure in one analysis. Once the subject classified the test word as visually or semantically dissimilar, processing could terminate and a response could be executed. For example, if the distractor were visually similar, more processing would perhaps have to be performed; that is, a semantic comparison made, before making a decision. Essentially this kind of a model assumes that added tests are rendered unnecessary when the first test detects dissimilarity of any kind.

A similar prediction can be made from the strength model, recently proposed by Atkinson, Herrmann and Wescourt (1974). Each item, when presented, elicits a 
level of familiarity. If this value falls below a low criterion, a decision is made to reject without further search. Similarly, if the value falls above a high criterion, a decision is made immediately to accept. If the elicited value falls between the two criteria, a search of memory must ensue before a decision is made. The more similar a distractor to a target set, the more likely it is to elicit a familiarity value above the low criterion, thus requiring additional time before a correct rejection. This strength model predicts, therefore, that items similar to the target set on physical and semantic dimensions will be rejected more slowly than items similar on only one dimension; and those, in turn, will be rejected more slowly than items dissimilar on both dimensions. This type of simple additive strength model predicts no interaction between physical and semantic variables.

The Atkinson and Juola version of the strength model makes similar predictions, but considers that the added time for processing visually similar distractors is needed in the encoding stage prior to any semantic analysis.

The following experiment investigated the separate and combined effects of visual similarity and semantic similarity between distractors and a target set on word recognition latency.

\section{METHOD}

\section{Subjects}

Subjects were nine female and seven male undergraduates from the University of Delaware. All subjects participated as a course requirement and had normal or corrected vision.

\section{Apparatus}

Words comprising the target set were printed on individual 89 x $159 \mathrm{~mm}$ white cards in black ink in $11 \mathrm{~mm}$ uppercase letters. These cards were used as stimuli during initial learning. During recognition testing, a three-channel Scientific Prototype tachistoscope (Model GB) was used to present a white prepost exposure field in one channel, a two-line fixation field in a second channel and the single-word probe displays in a third channel. Illumination for the fields and displays was $15 \mathrm{fc}$ measured at the eye. The probe words were typed in uppercase type, subtending a vertical visual angle of $0 \mathrm{deg} 11 \mathrm{~min}$ and, on the average, a horizontal angle of $1 \mathrm{deg} 18 \mathrm{~min}$. Due to an unavoidable equipment modification, eight subjects made the response by pressing microswitch buttons held between thumb and digits in each hand, and the other eight by pressing either of two predesignated adjacent keys with the index or middle finger of the right hand. Response times were recorded at least to $\pm 5 \mathrm{msec}$ accuracy with a Lafayette counter or clock timer.

\section{Procedure}

The subjects were tested individually in single sessions which were divided into a memorization period and a recognition test. At the beginning of a session, each subject was required to learn one of the two 40-word lists. Each list consisted of eight words from each of five categories: animals, articles of clothing, body parts, countries, and furniture. Each list also contained representatives from the same subcategories, e.g., bovines, a subcategory of animals, was represented by BULL on one list and BUFFALO on the other. The two lists were also matched on mean member frequency according to the Battig and Montague (1969) norms. The mean value for the two lists was approximately 93 in the norms. Finally, words were matched on visual similarity across lists, according to the following criteria: (1) Only words were used which began with one of the following letters: b, e, h, i, j, l, m, p, r, s, v, (2) all first letters were represented approximately equally on both lists, and (3) word length was equated at a mean value of 5.3 letters with a range of 3-8.

Each subject studied one list until he notified the experimenter that he was ready to attempt its recall. Additional time was given to the subject to study if he failed to recall the list perfectly. The average total study time was $20 \mathrm{~min}$. After the subject achieved $100 \%$ recall of the target list, he was given a recognition test. The test consisted of 80 single-word displays or probes. Forty probes were the target words which the subject had memorized and 40 probes were distractor words which, by definition, the subject had not memorized. There were four types of distractors: visually similar or visually dissimilar to the targets, and from a category on the target list or from a nontarget category. Each subject responded to 10 probes for each of the four distractor conditions of visually similar-target category, visually similar-nontarget category, visually dissimilar-target category, and visually dissimilar-nontarget category. Nontarget categories were chosen for their lack of any obvious conceptual similarity to target categories. These nontarget categories were: musical instruments, relatives, sports, units of time, and vehicles.

Visually similar-target category distractors were drawn from the list which subject had not memorized, since, as described above, the two lists were constructed with words which resembled the words on the other list visually and semantically. A constraint placed on the selection of this type of probe was that these words most resemble actual target words, i.e., not only were these words matched on first letter and length, but also when possible on other letters as well. The visually similar-nontarget category distractors were chosen to match targets in visual characteristics in the same manner as were the visually similar-target category distractors, e.g., if subject memorized EYE, a visually similar-target category distractor was EAR, and a visually similar-nontarget category distractor was EON. With three exceptions, words in the visually dissimilar conditions began with first letters not used in the target lists and were generally one or more letters shorter or longer than the mean length of the target words. In the case of the exceptions on first letter, these words were much longer or shorter than the average target, i.e., two or more letters greater or less than the mean target length. These exceptions were included in the stimulus pool, since it was impossible to find other words which came any closer in meeting all of the criteria for stimulus selection, i.e., the visual criteria, frequency in the Battig and Montague (1969) norms and, in the case of target category probes, a subcategory representation. Each visually dissimilar-target category probe was a member of a subcategory represented on the target list, e.g., for the category animals, the subcategory of bovines was represented in the visually dissimilar condition by $\mathrm{OX}$. The words in all distractor conditions were matched to the targets for mean frequency in the Battig and Montague (1969) norms.

Response type, as well as specific category membership of probes, was randomly ordered within blocks of 10 probes. In addition, distractors from all conditions were presented in each block.

During the recognition test, each trial was initiated by an auditory warning signal, followed by the two-line fixation field for $1.4 \mathrm{sec}$ and then by the probe word which remained in view until the subject responded. The subject was familiarized with the apparatus in eight practice trials which consisted of the subject pressing the appropriate button or key to a presentation of the probe words "yes" or "no." Then the subject had practice at making the recognition decision based on a sample target list of three vegetables. Distractors in these practice trials consisted of examples of each kind of condition. Instructions stressed 
accuracy over speed. Assignment of "yes" and "no" to dominant and nondominant hand or to index or middle finger was alternated across subjects.

In case of an error, subjects were asked immediately: (1) "Did you know you made an error?" (2) "What word did you think was in the display?" If a subject replied with an incorrect word, the response was labeled a misreading error. A complete session lasted from 1 to $1 \frac{1}{2} \mathrm{~h}$.

\section{RESULTS}

Median correct latencies were determined for each subject for each type of distractor and for targets. Figure 1 shows the means of median latencies for the four distractor conditions and for targets. It appears that rejection latencies to visually similar probes were greater than those to visually dissimilar probes and responses were shorter to nontarget category probes than to target category probes. In addition, there seems to be a strong interaction between the variables in that visually similar-nontarget category and visually dissimilar-nontarget category probes took the same time to reject. These impressions were supported statistically. Negative latency was affected significantly by visual similarity, $F(1,15)=29.5, p<.001$, and by category similarity, $F(1,15)=34.2, p<.001$. Interaction of visual and category similarity was significant, $F(1,15)=13.9$, $p<.01$. In order to test whether or not these significant effects are independent of the specific words used, Fs were computed collapsing across subjects and treating the population of words as random effect. The same pattern emerged, i.e., $F(1,76)=10.8, p<.01 ; F(1,76)=32.0$, $p<.001$; and $F(1,76)=6.3, p<.025$ for visual similarity, category similarity and their interaction, respectively. Minimum quasi Fs calculated according to Clark (1973) also substantiated the significance of the effects measured and the likelihood that results would generalize to the different sets of subjects and words. Minimum quasi $F$ s were: $F^{\prime}(1,84)=7.9, p<.01$; $F^{\prime}(1,52)=16.5, p<.001 ;$ and $F^{\prime}(1,78)=4.3, p<.05$ for visual similarity, category similarity and their interaction, respectively.

Target latency was significantly less than that for visually similar-target category distractors, $\mathrm{t}(15)=4.2$, $\mathrm{p}<.001$, and significantly longer than visually similar-nontarget category distractors, $t(15)=2.9$, $\mathrm{p}<.02$, and visually dissimilar-nontarget category distractors, $t(15)=3.3, p<.01$.

The error rate was $1.8 \%$ of all responses, of which $0.8 \%$ were to target probes and $1.0 \%$ to distractors. The incorrect responses to distractors included $0.9 \%$ to visually similar-target category probes, $0.1 \%$ to visually similar-nontarget category probes, and $0 \%$ to visually dissimilar-target category and visually dissimilar-nontarget category probes. Nine misreading errors were made by seven subjects; and except for one instance, these errors were target words given to visually similar distractors.

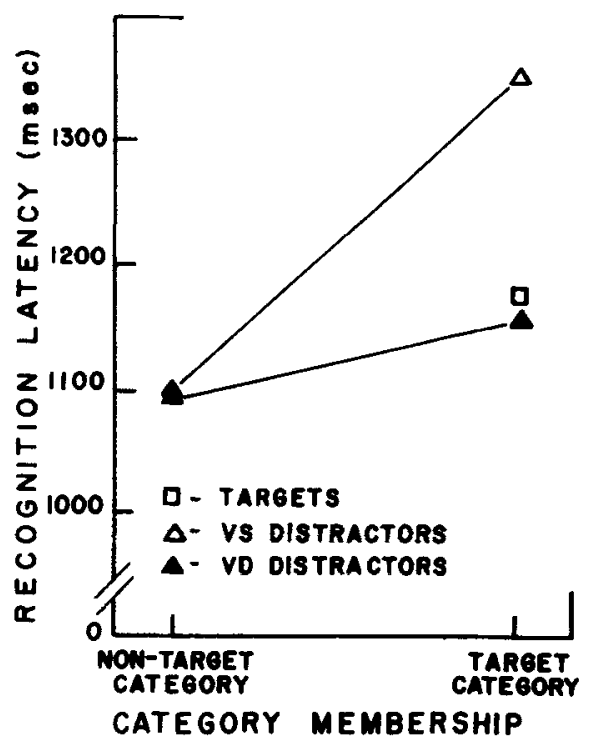

Figure 1. Recognition latencies for targets and for distractors which were visually similar (VS) and dissimilar (VD) to targets and which belonged to target categories and to nontarget categories.

\section{DISCUSSION}

The study clearly confirms the earlier findings of Juola et al. (1971) and of Okada and Burrows (1971) that similarity of shape and of semantic features between targets and distractors affects rejection latency. Perhaps more importantly, the present study demonstrates that the variables interact.

The interaction suggests that the decision to reject can be made after the first termination of an analysis of the input. The virtual identity of the latencies in the visually dissimilar-nontarget category and visually similar-nontarget category conditions suggests that the semantic analysis may be the first one which is completed. The shape analysis may begin after the semantic or it may occur in parallel, taking a longer time.

Wickens (1973) suggested that certain attributes of a word may be processed at different rates and gives evidence for immediate and quick semantic analysis even before the naming or identification is completed. Posner and Warren (1973) also stress such encoding processes can proceed in parallel. If semantic analysis finishes first, the distractor can be rejected even before the probe is identified. Only those distractors that have passed the semantic analysis will show the effect of added time needed for being visually similar. Our data give support to these ideas of parallel encoding of semantic and visual attributes, with the semantic encoding finishing first.

The strength models proposed by Atkinson and his associates cannot explain the interaction without more elaboration. Word shape seemed to affect familiarity of target category distractors only. Distractors that were 
unrelated to targets were rejected quickly whether or not they looked like the targets. If, as Atkinson and Juola (1972) suggest, shape has its effect before semantic processing, then visually similar distractors should have required more time than the visually dissimilar whether or not they belonged to target categories. Or if, as Atkinson et al. (1974) suggest, shape and meaning affect familiarity judgments, then no interaction should have resulted.

Some support for the idea that visual similarity between target and distractor leads to confusion and misidentification (Juola et al., 1971) can be found in the error data. All 13 distractor errors were in visually similar conditions, and eight of these distractor errors were reported as actual misreadings. However, these data do not indicate whether identification comes before or after the semantic processing.

\section{REFERENCES}

Atkinson, R. C., Hermann, D. J., \& Wescourt, K. T. Search processes in recognition memory. In R. L. Solso (Ed.) Theories in cognitive psychology: The Loyola Symposium. Potomac, Md: Lawrence Erlbaum Associates, 1974.
Battig, W. F., \& Montague, W. E. Category norms for verbal items in 56 categories; a replication and extension of the Connecticut category norms, Journal of Experimental Psychology, 1969, 80, (3. Pt. 2).

Clark, H. H. The language-as-fixedeffect fallacy: A critique of language statistics in psychological research. Journal of Verbal Learning and Verbal Behavior, 1973, 12, 335-339.

Juola, J. F., Fischler, I. S., Wood, C. T., \& Atkinson, R. C. Recognition time for information stored in long-term mem ory. Perception \& Psychophysics. 1971. 10. 8-14.

Neisser, U., \& Beller, H. K. Searching through word lists, British Journal of Psychology, 1965, 56, 349-358.

Posner, M. I., \& Warren, R. E. Traces, concepts and conscious constructions. In A. W. Melton and E. Martin (Eds.) Coding processes in human memory. Washington, D.C: V. H. Winston, 1972.

Wickens. D. D. Characteristics of word encoding. In A. W. Melton and E. Martin (Eds.) Coding processes in human memory. Washington, D.C: V. H. Winston, 1972.

\section{NOTES}

1. Atkinson, R. C., \& Juola, J. F. Search and decision processes in recognition memory. Technical Report No. 194, 1972. Stanford University, Institute for Mathematical Studies in the Social Sciences, Stanford, California.

2. Okada, R., \& Burrows, D. Organizational factors in high-speed memory search. Paper read at the Eastern Psy chological Association. New York City, April 1971.

(Received for publication July 7, 1974: revision received September $27,1974$. ) 